\title{
Low Serum Hepatitis B Surface Antigen Level Predicts Compensated Cirrhosis Caused by Chronic Hepatitis B in HBeAg Positive Patients in East China
}

\author{
Wen Jia ${ }^{1,2}$; Xun Qi ${ }^{1}$; Yan-Yan Ji ${ }^{2}$; Yun-Hao Xun ${ }^{3}$; Hong Wang ${ }^{2}$; Wen-Hong Zhang ${ }^{1,2}$; Jian-Hua \\ Yang $^{2}$; Jin-Yu Wang ${ }^{1}$; Hao-Xiang Zhu ${ }^{1}$; Ri-Cheng Mao ${ }^{1, *}$; Ji-Ming Zhang ${ }^{1,2,}$ \\ ${ }^{1}$ Department of Infectious Diseases, Huashan Hospital, Fudan University, Shanghai, China \\ ${ }_{3}^{2}$ Department of Infectious Diseases, Jing'An District Centre, Shanghai Hospital, Fudan University, Shanghai, China \\ ${ }^{3}$ Department of Liver Diseases, Xixi Hospital of Hangzhou, Zhejiang Chinese Medical University, Hangzhou, China \\ ${ }^{*}$ Corresponding Authors: Ji-Ming Zhang, Department of Infectious Diseases, Huashan Hospital, Fudan University, Shanghai, China. Tel: +86-2152887963, Fax: +86-2152886140, \\ E-mail: jmzhang@fudan.edu.cn; Ri-Cheng Mao, Department of Infectious Diseases, Huashan Hospital, Fudan University, Shanghai, China. Tel: +86-2152887963, Fax: +86-2152886140, \\ E-mail: richengmao@gmail.com
}

Received: April 12, 2015; Revised: May 27, 2015; Accepted:July 7, 2015

Backgrounds: Serum hepatitis B surface antigen (HBsAg) levels are associated with fibrosis in patients with chronic hepatitis B (CHB) infection.

Objectives: The aim of our study was to evaluate serum HBsAg level as a biomarker for compensated cirrhosis in hepatitis B e antigen (HBeAg) positive CHB patients.

Patients and Methods: Two-hundred and one HBeAg-positive Chinese CHB patients with or without cirrhosis were enrolled in this retrospective study. Cirrhosis was diagnosed based on liver biopsy. Furthermore, patients with decompensated cirrhosis were excluded. A statistical analysis was performed regarding the association between serum HBsAg level and compensated cirrhosis.

Results: Patients with compensated cirrhosis had a significantly lower mean serum HBsAg level compared to those without cirrhosis (3.27 $\log _{10} \mathrm{IU} / \mathrm{mL}$ VS $4.17 \log _{10} \mathrm{IU} / \mathrm{mL}, \mathrm{P}<0.001$ ). Furthermore, examining the correlation with compensated cirrhosis revealed that lower level of serum HBsAg was a significant factor in multivariate analysis. The area under the receiver operating characteristics curve of serum HBsAg was 0.856 for compensated cirrhosis. A positive predictive value of $66.2 \%$ and negative predictive value of $90.7 \%$ were obtained with a cut-off value of $<3.60 \log _{10} \mathrm{IU} / \mathrm{mL}(4000 \mathrm{IU} / \mathrm{mL})$ of serum HBsAg. Moreover, the rate of compensated cirrhosis increased to $75.0 \%$ after combining with APRI $>2$.

Conclusions: In HBeAg positive CHB patients, low serum HBsAg level is a useful predictor of compensated cirrhosis.

Keywords: Hepatitis B Surface Antigens; Liver Cirrhosis; Hepatitis B e Antigens; Hepatitis B; Chronic

\section{Backgrounds}

Liver cirrhosis is a leading cause of death in patients with chronic hepatitis B (CHB) and can lead to portal hypertension, which causes end-stage liver diseases such as oesophageal variceal bleeding (1) and hepatic encephalopathy (2). The 5-year survival rate was $80 \%-85 \%$ in patients with compensated cirrhosis, while $14 \%$ - 35\% in patients with decompensated cirrhosis $(3,4)$. Since antiviral therapy can reduce progression of cirrhosis and prevent hepatic decompensation in patients with compensated cirrhosis (5-7), an early diagnosis of compensated cirrhosis is critical for improving patient prognosis.

The specificity of ultrasonography, computed tomography (CT) and magnetic resonance imaging (MRI) to detect cirrhosis is high. Imaging reveals an inhomogeneous hepatic texture or surface, a rarefied hepatic central vein, an enlarged caudate lobe and splenomegaly or collateral veins. However, these imaging modalities are not sensi- tive enough to detect cirrhosis (8) and liver biopsy is still the gold standard for diagnosis.

Given the invasive nature of liver biopsy, several noninvasive indices have been developed for prediction of cirrhosis in CHB (9-11). Transient electrograph (FibroScan) is a method for assessing liver cirrhosis, but is limited due to its expensive cost (12). Thus, WHO suggested to use aminotransferase/platelet ratio index (APRI) for the assessment of liver cirrhosis in developing countries (13). Nonetheless, APRI has only a moderate diagnostic accuracy (14-16). Therefore, it is reasonable to explore other serum-based noninvasive tests to predict compensated cirrhosis.

Recent studies revealed that serum hepatitis B surface antigen (HBsAg) levels are inversely correlated with fibrosis scores $(17,18)$ and high serum HBsAg levels can identify insignificant fibrosis in treatment-naive hepatitis B e

Copyright (C) 2015, Kowsar Corp. This is an open-access article distributed under the terms of the Creative Commons Attribution-NonCommercial 4.0 International License (http://creativecommons.org/licenses/by-nc/4.0/) which permits copy and redistribute the material just in noncommercial usages, provided the original work is properly cited. 
antigen (HBeAg)-positive hepatitis B virus (HBV) carriers (19-22). Due to the above reasons, serum HBsAg levels may be correlated with liver cirrhosis caused by CHB.

\section{Objectives}

In this study, we aimed to determine the performance of serum HBsAg level as a biomarker for compensated cirrhosis in HBeAg-positive CHB patients.

\section{Patients and Methods}

\subsection{Patients}

Patients with HBeAg-positive CHB and treatment-naive were included in this retrospective study. Patients were recruited from Huashan Hospital, Fudan University and Xixi Hospital of Hangzhou, Zhejiang Province during June 2007 to May 2014. All patients were HBsAg and HBeAg positive for at least six months before enrollment in the study. All patients underwent liver biopsy. Exclusion criteria were concomitant liver diseases including chronic hepatitis C or D infection, Wilson's disease, autoimmune hepatitis and primary biliary cirrhosis. Patients with decompensated cirrhosis or those consuming a sig nificant amount of alcohol were excluded (30 g/day for men, $20 \mathrm{~g}$ /day for women) as well. In addition, patients with inadequate serum for analysis or different opinions on liver biopsy were excluded. According to the inclusion and exclusion criteria, 201 patients were included and 123 patients excluded in our research. Our sample size achieved the expected power. Flowchart of study participants is shown in Figure 1.

The study was approved by the ethical committee of Huashan Hospital (200705) and Xixi Hospital of Hangzhou (201309). All subjects gave informed consent for their participation in the study.

\subsection{Clinical and Laboratory Assessment}

Serum samples were taken at baseline and stored at $-20^{\circ} \mathrm{C}$. All samples were tested in the Huashan Hospital laboratory. Relevant clinical variables included age, sex, serum alanine aminotransferase (ALT), aspartate aminotransferase (AST), albumin (A), and globulin (G) levels, platelet (PLT) counts. ALT, AST, A and total protein levels were measured in UniCel Dxc 800 using Synchron system Kits (Beckman Coulter), and G levels were determined by subtracting the amount of albumin levels from the total protein levels. In addition, PLT counts were measured in Sysmex XT-1800i using its corresponding commercially available kit (Sysmex Corporation). Serum HBV DNA levels were measured in Lightcycle using the COBAS TaqMan assay (Roche Diagnostics), with a linear range of $20-1.98 \times 10^{8} \mathrm{IU} / \mathrm{mL}$. Samples with HBV DNA levels higher than $1.98 \times 10^{8} \mathrm{IU} /$ $\mathrm{mL}$ were diluted at 1:100 for retesting.

Serum HBsAg, HBeAg and antibody to hepatitis B e anti- gen (anti-HBe) were measured using commercially available immunoassays (Abbott Laboratories, Chicago, IL). Serum HBsAg level detection value ranged from 0.05 to $250 \mathrm{IU} / \mathrm{mL}$ and samples with an HBsAg level $>250 \mathrm{IU} / \mathrm{mL}$ required a 1:500 or greater dilution. All the tests above are subject to strict quality control. Furthermore, our laboratory passed the certificate from the College of American Pathologists (CAP).

AST to platelet ratio index (APRI) was evaluated for grading the extent of liver fibrosis. APRI $=($ AST $[$ IU $/ L]) /($ ULN, 40 at our laboratory $) /\left(\right.$ PLT $\left.\left[10^{9} / \mathrm{L}\right]\right) \times 100(23)$.

\subsection{Liver Histology}

Liver biopsy was performed using a 16-gauge Menghini needle and specimens were fixed in formalin and embedded in paraffin. Hematoxylin-eosin and reticular fibre staining or Masson staining were performed on each section. Two experienced pathologists examined the sections independently. Grading of inflammation $(G)$ and staging of fibrosis $(S)$ were determined using the modified Scheuer scoring system (24) and scored as G0 - G4 and So - S4, respectively. A stage of S4 represented liver cirrhosis. If there were different opinions on the stage of liver biopsy, the patient was excluded.

Figure 1. Flowchart of Study Participants

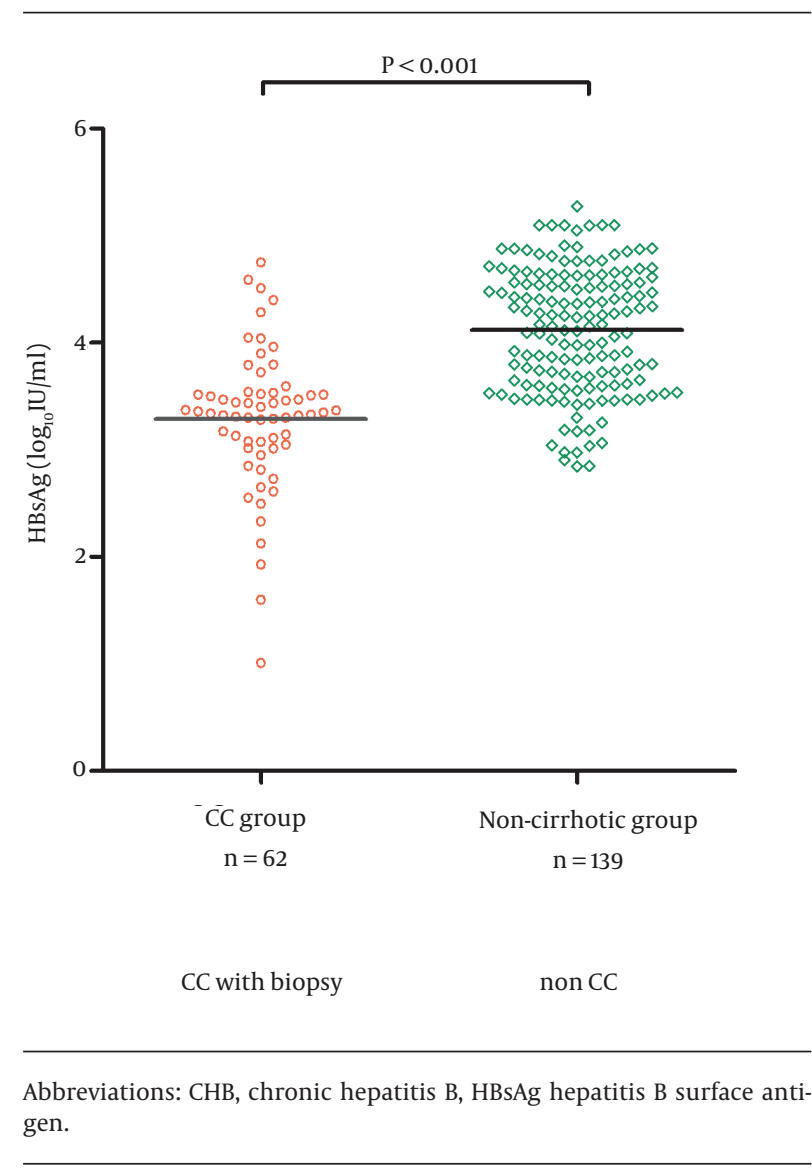


Jia Wet al.

\subsection{Statistical Analyses}

All data were processed using Stata 10.0 software (Stata Corporation, College Station, TX, USA). Shapiro-wilk test was used to verify the normal assumption. Parametric variables were expressed as mean \pm standard deviation (SD) and nonparametric were expressed as median (P25, P75), assessed by Student t-test (parameter) or MannWhitney (non-parameter) test as appropriate. Categorical variables were analyzed using the chi-square test. Spearman's correlation coefficient ( $r$ ) was used for correlation analysis. Additionally, independent factors associated with compensated cirrhosis were identified using multivariate logistic regression analysis. Furthermore, area under receiver-operator curve (AUC) was used to calculate the diagnostic accuracy of serum HBsAg and APRI and detailed information including sensitivity, specificity, positive predictive values (PPVs) and negative predictive values (NPVs) for relevant cut-offs were also displayed. The Youden index, defined as sensitivity plus specificity minus one, was used to identify the optimal level of prediction. A two-sided P value of 0.05 was considered statistically significant.

\section{Results}

\subsection{Patients' Characteristics}

Baseline demographics of patients are shown in Table 1.
Only three patients with compensated cirrhosis were classified in Child-Pugh class B and the remainder were in class A. Patients with compensated cirrhosis were categorized as CC group $(n=62)$ and those without cirrhosis were categorized as non-cirrhotic group $(n=139)$. All of the variables were nonparametric. Nevertheless, when we changed HBV DNA and HBsAg to $\log _{10}$ HBV DNA and $\log _{10}$ HBsAg, they both accorded with normal distribution. Comparison of patients with CC group and non-cirrhotic group showed that patients in the CC group were older, had lower ALT, albumin and PLT levels, higher AST levels and lower HBsAg and HBV DNA levels ( $\mathrm{P}<0.05)$. In our research, 24 patients had fibrosis stage So, and 38, 43, 34, 62 patients had stages S1, S2, S3, S4, respectively. In addition, nine patients had inflammation grading Go and 31, 39, 66, 56 patients had G1, G2, G3, G4, respectively. There was a strong inverse correlation between serum HBsAg and fibrosis $(\mathrm{r}=-0.668, \mathrm{P}<$ 0.001), while no correlation between serum HBsAg and inflammation $(\mathrm{r}=-0.129, \mathrm{P}=0.201)$ (Figure 2 ).

\subsection{Serum HBsAg Levels for CC Group Were Signifi- cantly Lower Than Those for Non-Cirrhotic Group}

Serum HBsAg level $\left(\log _{10} \mathrm{IU} / \mathrm{mL}\right)$ for the CC group and non-cirrhotic group were $3.27 \pm 0.71$ and $4.17 \pm 0.57(\mathrm{P}<$ 0.001), respectively (Figure 3 ). Serum HBsAg levels were significantly lower for CC group compared to non-cirrhotic group.

Table 1. Baseline Characteristics of 201 Patients ${ }^{a}$

\begin{tabular}{|c|c|c|c|}
\hline Variables & CC Group b & Non-Cirrhotic Group & P Value \\
\hline Number of patients & 62 & 139 & - \\
\hline Age, $y^{b}$ & $37.0(28.0,42.0)$ & $30.5(25.3,39.0)$ & $<0.001$ \\
\hline Male patients ${ }^{\mathrm{C}}$ & $47(77)$ & $114(82)$ & 0.078 \\
\hline $\mathbf{A L T}, \mathbf{U} / \mathbf{L}^{\mathrm{b}}$ & $85(37.0,240.8)$ & $94(48.8,189.5)$ & 0.014 \\
\hline AST, $\mathbf{U} / \mathbf{L}^{\mathrm{b}}$ & $51.5(39.7,64.9)$ & $46(36.4,56.2)$ & 0.005 \\
\hline PLT, $\times$ T9/L L $^{b}$ & $112.8 \pm 55.6$ & $168.8 \pm 50.7$ & 0.017 \\
\hline $\mathbf{A}, \mathbf{g} / \mathbf{L}^{\mathrm{b}}$ & $42.4(36.0,43.0)$ & $46.4(44.0,48.0)$ & $<0.001$ \\
\hline $\mathbf{G}, \mathbf{g} / \mathbf{L}^{\mathrm{b}}$ & $31.4 \pm 5.8$ & $32.4 \pm 5.3$ & 0.311 \\
\hline HBV DNA, $\log _{10} I U / \mathrm{mL}^{\mathrm{d}}$ & $6.77 \pm 1.70$ & $7.49 \pm 1.33$ & 0.032 \\
\hline HBsAg, $\log _{10} I U / m^{d}$ & $3.27 \pm 0.71$ & $4.17 \pm 0.57$ & $<0.001$ \\
\hline
\end{tabular}

a Abbreviations: A, serum albumin; ALT, alanine aminotransferase; AST, aspartate aminotransferase; CC group, patients with compensated cirrhosis; G, serum globulin; HBsAg, hepatitis B surface antigen; and PLT, platelet count.

b Nonparametric variables are expressed as median (P25, P75).

c Categorical variables are expressed as No. (\%).

$\mathrm{d}$ Parametric variables are expressed as median mean \pm standard deviation. 
Jia Wet al.

4.3. Univariate and Multivariate Analysis for Predicting Compensated Cirrhosis

The univariate and multivariate analyses of factors predictive of cirrhosis in HBeAg positive $\mathrm{CHB}$ patients are shown in Table 2. Older age, lower platelet count, lower albumin level, higher AST level, lower HBsAg and HBV DNA level were all associated with compensated cirrhosis in the univariate analysis.

After adjusting for different clinical parameters, independent factors associated with compensated cirrhosis included lower albumin level (OR 0.938; 95\% CI 0.894 to 0.990; $\mathrm{P}=0.011$ ), lower serum HBsAg level (OR 0.100; 95\% CI 0.037 to 0.272; P<0.001) and lower PLT count (OR 0.973; 95\% CI 0.959 - 0.986; $\mathrm{P}<0.001$ ) (Table 2).

\subsection{Predictive Value of Serum HBsAg for Differen- tiating CHB Patients With Compensated Cirrhosis From Those Without Cirrhosis}

As an independent factor associated with compensated cirrhosis, the ROC curves of serum HBsAg levels in predicting compensated cirrhosis are depicted in Figure 4 (ROC, 0.856; Standard Error, 0.028, P < 0.001; 95\% confidence interval, 0.799 - 0.914). Based on the Youden Index, the optimal cutoff level of serum HBsAg to predict CC was 3.60 $\log _{10} \mathrm{IU} / \mathrm{mL}$ (4000 IU/mL), which was able to predict compensated cirrhosis with a sensitivity of $80.3 \%$, specificity of $82.4 \%$, positive predictive value of $66.2 \%$ and negative predictive value of $90.7 \%$. Serum HBsAg $<500 \mathrm{IU} / \mathrm{mL}$ (2.70 $\log _{10} \mathrm{IU} / \mathrm{mL}$ ) was $100 \%$ predictive of compensated cirrhosis. For the purpose identifying but not excluding cirrhosis, thereby reciprocal of the serum HBsAg (1/HBsAg) was made in ROC curves.
Figure 2. The Differences in HBsAg Serum Levels for Different Stages of Fibrosis

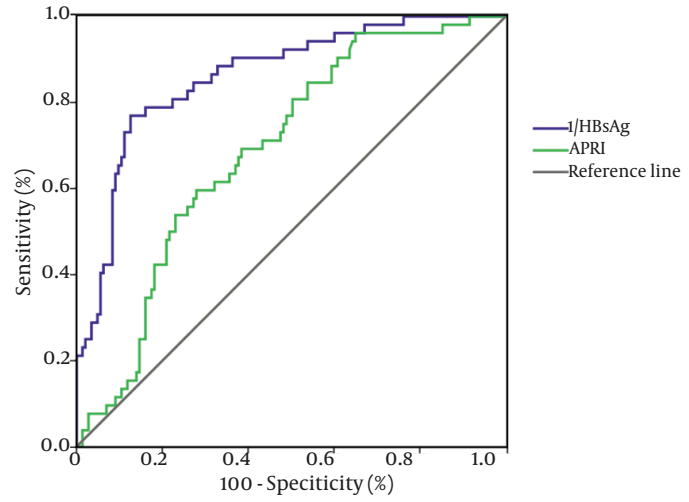

Serum HBsAg were expressed as mean \pm standard deviation, the result showed that serum HBsAg decreased with the process of fibrosis. There was a strong inverse correlation between serum HBsAg and fibrosis $(r=$ $-0.668, \mathrm{P}<0.001)$. Abbreviations: HBsAg, hepatitis B surface antigen.

Figure 3. The Differences in HBsAg Serum Levels Between CC Group and Non-Cirrhotic Group

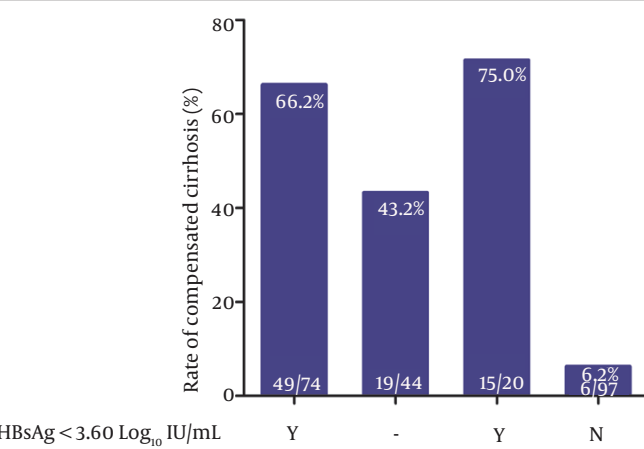

APRI $>2$

CC group: patients with compensated cirrhosis; non-cirrhotic group: patients without cirrhosis.

\begin{tabular}{|c|c|c|c|c|c|c|}
\hline \multirow[t]{2}{*}{ Variables } & \multicolumn{3}{|c|}{ Univariate Analysis } & \multicolumn{3}{|c|}{ Multivariate Analysis } \\
\hline & Coefficient & OR, 95\% CI & P Value & Coefficient & OR, 95\% CI & P Value \\
\hline Gender (male) & 0.138 & $1.148(0.534-2.468)$ & 0.723 & 0.123 & $1.131(0.296-4.328)$ & 0.857 \\
\hline Age, $y$ & 0.029 & $1.029(1.001-1.057)$ & 0.039 & 0.004 & $1.003(0.960-1.050)$ & 0.868 \\
\hline HBsAg, $\log _{10} \mathrm{IU} / \mathrm{mL}$ & -2.358 & $0.095(0.045-0.200)$ & $<0.001$ & -2.300 & $0.100(0.037-0.272)$ & $<0.001$ \\
\hline ALT, U/L & -0.001 & $0.998(0.996-1.001)$ & 0.140 & -0.001 & $0.999(0.996-1.002)$ & 0.481 \\
\hline AST, $U / L$ & 0.330 & $1.391(1.083-1.788)$ & 0.010 & 0.003 & $1.012(1.001-1.061)$ & 0.414 \\
\hline HBV DNA, $\log _{10} \mathrm{IU} / \mathrm{mL}$ & -0.237 & $0.789(0.633-0.983)$ & 0.035 & -0.043 & $0.897(0.609-1.127)$ & 0.862 \\
\hline PLT, $\times$ T9 $/$ L a & -0.008 & $0.992(0.984-0.999)$ & $<0.001$ & -0.028 & $0.973(0.959-0.986)$ & $<0.001$ \\
\hline Albumin, $\mathrm{g} / \mathrm{L}$ & -0.187 & $0.861(0.800-0.921)$ & $<0.001$ & -0.012 & $\begin{array}{c}0.938(0.894- \\
0.990)\end{array}$ & 0.011 \\
\hline
\end{tabular}

a Abbreviations: 95\% CI, 95\% confidence interval; ALT, alanine aminotransferase; AST, aspartate aminotransferase; HBsAg, hepatitis B surface antigen; $\mathrm{HBV}$, hepatitis B virus; OR, odds ratio; and PLT, platelet. 


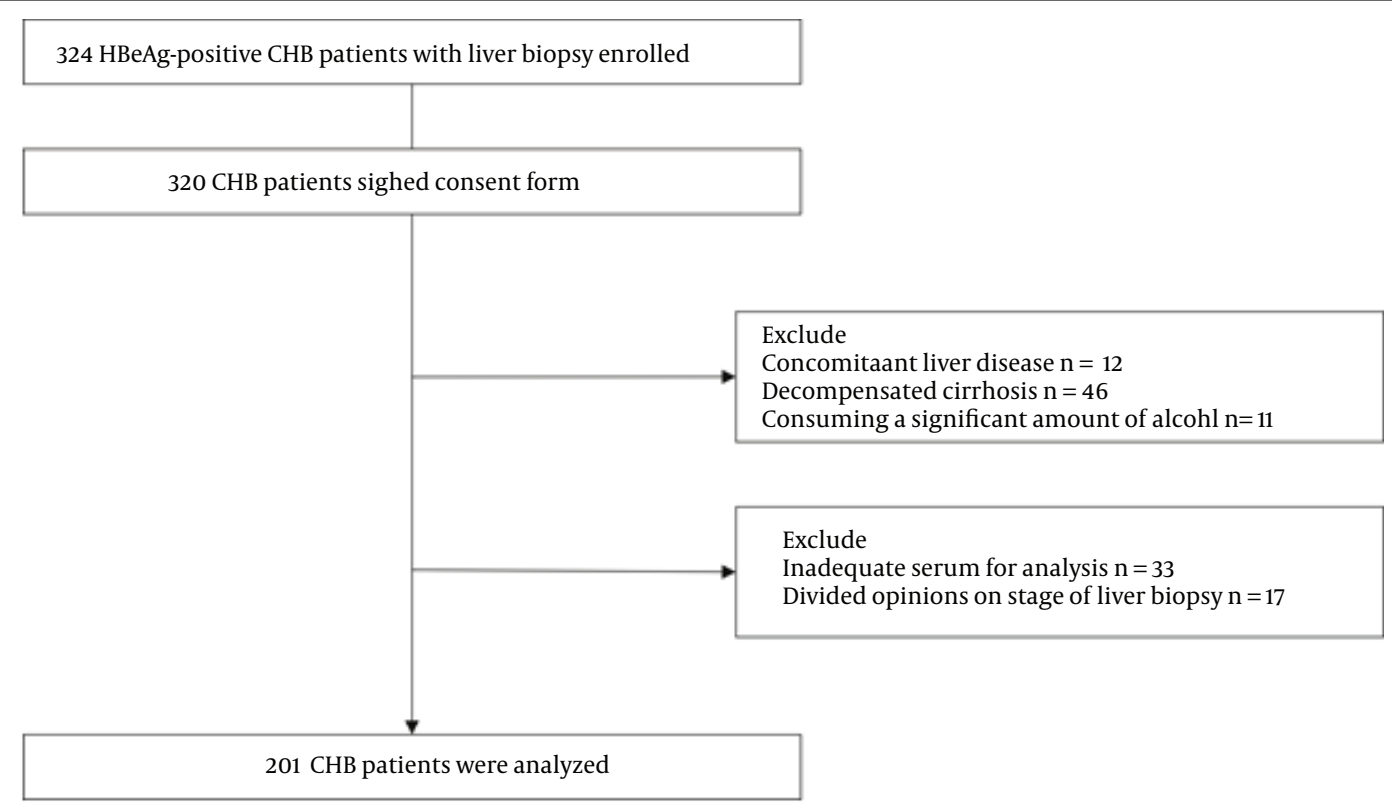

Abbreviations: HBsAg, hepatitis B surface antigen; and APRI, aspartate aminotransferase to platelet ratio index.

\subsection{Combining HBsAg With APRI Could Better Pre- dict Compensated Cirrhosis}

Previous studies had identified APRI as a predictor of cirrhosis. In the current study, the ROC curves of APRI in predicting compensated cirrhosis are depicted in Figure 4 (ROC, 0.699; Standard Error, 0.038, P < 0.001; 95\% confidence interval $0.625-0.772$ ). $43.2 \%$ of patients with APRI more than 2 had compensated cirrhosis (Figure 5). Moreover, we examined the rates of compensated cirrhosis among patients with HBsAg below $3.6 \log _{10} \mathrm{IU} / \mathrm{mL}$ and APRI more than 2 and taking into account their proportion of compensated cirrhosis. The results showed that among the above subgroups, 75.0\% (15/20) achieved compensated cirrhosis (Figure 5).

\section{Discussion}

Although liver biopsy is a gold standard for the diagnosis of liver cirrhosis, it is an expensive and invasive procedure associated with patient discomfort, a small risk of serious bleeding and requires specialist histological examination for accurate staging (13). Therefore, many noninvasive methods have been applied to substitute this invasive procedure. We performed this study in a treatment-naive Chinese cohort of HBeAg-positive CHB patients. Serum HBsAg was analyzed in patients with or without cirrhosis and was evaluated as a predictive marker of compensated cirrhosis. We found a significantly lower level of HBsAg in cirrhotic group. To compare HBsAg with other predictors, we conducted a multivariate regression analysis. The results indicated that $\mathrm{HBsAg}$ level could predict compensated cirrhosis independently (OR 0.100; $\mathrm{P}<0.001$ ).
Figure 5. Rate of Compensated Cirrhosis Among Subgroups of Patients Stratified by HBsAg and APRI

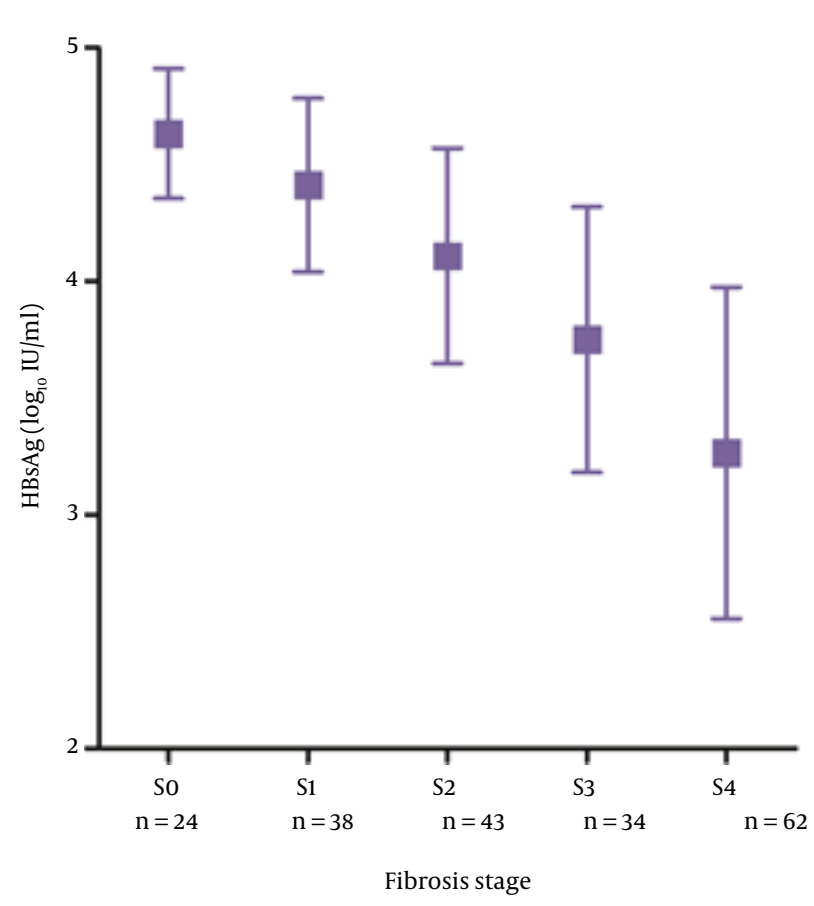

Abbreviations: APRI, aspartate aminotransferase to platelet ratio index; and HBsAg, hepatitis B surface antigen. 
In addition, PLT count and albumin level were independently related to compensated cirrhosis as expected, which were similar with previous studies $(25,26)$.

In our study, the optimal cut-off level of serum HBsAg to predict cirrhosis was $3.60 \log _{10} \mathrm{IU} / \mathrm{mL}(4000 \mathrm{IU} / \mathrm{mL})$, which may be used to distinguish compensated cirrhotic patients from non-cirrhotic patients. Our results suggest that HBeAg-positive patients with HBsAg below 3.60 $\log _{10} \mathrm{IU} / \mathrm{mL}$ ( $4000 \mathrm{IU} / \mathrm{mL}$ ) were at high risk of developing compensated cirrhosis. Moreover, serum HBsAg below 2.70 $\log _{10} \mathrm{IU} / \mathrm{mL}$ (500 IU/mL) was $100 \%$ predictive of compensated cirrhosis in our research group. Thus, HBeAgpositive patients with HBsAg below 2.70 $\log _{10} \mathrm{IU} / \mathrm{mL}$ (500 $\mathrm{IU} / \mathrm{mL}$ ) are more likely to have compensated cirrhosis even with no evidence in imaging scan.

In previous studies, several indices have been proposed as potential noninvasive predictors of cirrhosis in $\mathrm{CHB}$ patients, including FIB-4, APRI and FibroScan. FIB-4 index, first characterized in an HIV-HCV co-infected population and subsequently evaluated in HCV-infected subjects, may perform favorably to determine the extent of fibrosis in patients with $\mathrm{CHB}$ (27). Nonetheless, it has been developed and validated for detection of fibrosis stages $\geq$ S3 but not cirrhosis $(13,28)$. APRI is based on two indirect markers of fibrosis, a single high cut-off more than 2 is used for identifying patients with cirrhosis. However, an APRI score more than 2 could detect only one third of patients with cirrhosis (29). FibroScan had a relatively high AUROC in predicting cirrhosis compared to APRI while is limited by its high cost (30). In addition, the expenses for preventive, corrective maintenance and trained operators are high. Thus, the use of FibroScan is limited in developing countries. Therefore, the new WHO treatment guideline for HBV recommended that APRI could be used to predict cirrhosis (13) in developing countries.

In our research, we evaluated the predictive value among subgroups of patients stratified by APRI and serum HBsAg. The results showed that the rate of compensated cirrhosis increased from $66.2 \%$ to $75.0 \%$ after combining the APRI $>2$ and $\mathrm{HBsAg}<3.6 \log _{10} \mathrm{IU} / \mathrm{mL}$. The above results indicated that APRI combined with HBsAg could further improve the predictive value to some extent.

The mechanism underlying the predictive value of HBsAg is still unknown. Many studies have shown that serum HBsAg level decreased with fibrosis progress (20-22, 31), which were similar with our finding (CC group 3.27 $\log _{10} \mathrm{IU} / \mathrm{mL}$ VS. non-cirrhotic group 4.17 $\log _{10} \mathrm{IU} / \mathrm{mL}, \mathrm{P}<$ 0.001). The mechanism of the association between HBsAg and cirrhosis may be related to the fact that patients with cirrhosis have a diminished ability to support viral replication (18). Moreover, Cheng et al. (17) concluded that during disease escalation, active host immunity would be triggered against HBV replication and possibly results in chronic liver injury, which leads to decrease of HBsAg and develop of liver fibrosis. In addition, Pollicino et al. (32) demonstrated that preS/S HBV mutants were positively correlated with cirrhosis, which decreased the level of serum HBsAg.

It is advised that CHB patients with compensated cirrhosis should be treated to prevent further clinical events, even if the HBV DNA level is low or undetectable (13). Serum HBsAg measuring is less expensive, largely automatic and requires only phlebotomy. According to our results, HBsAg combined with APRI could further improve the predictive value for detecting compensated cirrhosis. Nonetheless, our study still has some limitations. Firstly, information on HBV genotypes was not available. Whereas, the major HBV genotypes in China are B and C, which are virtually indistinguishable for HBsAg expression pattern (33) and have similar prediction of fibrosis severity $(21,34)$. Secondly, our research was a retrospective study, which was prone to bias. Therefore, randomized prospective studies with larger sample size are needed to validate our results.

In conclusion, patients with compensated cirrhosis caused by CHB have significantly lower HBsAg levels compared to those without cirrhosis. Serum HBsAg level less than $3.60 \log _{10} \mathrm{IU} / \mathrm{mL}(4000 \mathrm{IU} / \mathrm{mL})$ may serve as a noninvasive candidate biomarker for compensated cirrhosis. Thus, this special population should be closely monitored to receive proper treatment timely.

Table 3. Sensitivity, Specificity and Predictive Values of Different HBsAg Levels for Predicting Compensated Cirrhosis a

\begin{tabular}{|c|c|c|c|c|c|c|c|}
\hline HBsAg, IU/mL & Number of Patients & Sensitivity ${ }^{\mathrm{b}}$ & Specificity ${ }^{b}$ & $\mathbf{P P V}^{\mathrm{b}}$ & $\mathbf{N P V}^{\mathrm{b}}$ & $\mathbf{L R}+$ & LR- \\
\hline$<4000,3.60 \log _{10}$ & 74 & 80.3 & 82.4 & 66.2 & 90.7 & 4.56 & 0.22 \\
\hline$<3000,3.48 \log _{10}$ & 60 & 67.2 & 86.6 & 68.3 & 86.1 & 5.02 & 0.38 \\
\hline$<2000,3.30 \log _{10}$ & 38 & 42.6 & 91.5 & 68.4 & 78.8 & 5.04 & 0.63 \\
\hline$<1000,3.00 \log _{10}$ & 18 & 21.3 & 96.4 & 72.2 & 74.1 & 6.05 & 0.82 \\
\hline$<500,2.70 \log _{10}$ & 9 & 14.8 & 100 & 100 & 73.2 & - & 0.85 \\
\hline
\end{tabular}

a Abbreviations: HBsAg, hepatitis B surface antigen; LR+, positive likelihood rate; LR-, negative likelihood rate; NPV, negative predictive value; and PPV, positive predictive value.

balues' unit is \%. 


\section{Acknowledgements}

We thank Dr. Xiao-Qin Wang (Department of Hematology, Fudan University) for their helpful suggestions during the study.

\section{Authors' Contributions}

Wen Jia, Ri-Cheng Mao and Ji-Ming Zhang were responsible for the study design and data analysis. Wen Jia, Yun-Hao Xun, Hong Wang and Xun Qi were responsible for patient recruitment and care. Xun Qi and Wen-Hong Zhang were responsible for histological assessment and fibrosis scoring. Jian-Hua Yang and Hao-Xiang Zhu were responsible for clinical and laboratory assessment. Xun Qi and Yan-Yan Ji were responsible for database management. All authors contributed to the final version of the manuscript.

\section{Funding/Support}

This research was funded in part by grants from the Shanghai Health Bureau (Young Program, 20114Y088), the National Natural Science Foundation of China (81201277 and 81271833), the "973" Project (2012CB519001), the Elite Doctor Training Programme of Jing'An District (JWRC 2014G06), the Elite Yong Doctors Cultivation Programme of Shanghai and Major Science and Technology Special Project of China grants (2012ZX10002007-001-002 and 2013ZX10002001).

\section{References}

1. Garcia-Tsao G. Current management of the complications of cirrhosis and portal hypertension: Variceal hemorrhage, ascites, and spontaneous bacterial peritonitis. Gastroenterology. 2001;120(3):726-48.

2. Butterworth RF. Complications of cirrhosis III. Hepatic encephalopathy. J Hepatol. 2000;32(1 Suppl):171-80.

3. Chu CM, Liaw YF. Hepatitis B virus-related cirrhosis: natural history and treatment. Semin Liver Dis. 2006;26(2):142-52.

4. Fattovich G. Natural history and prognosis of hepatitis B. Semin Liver Dis. 2003;23(1):47-58.

5. European Association For The Study Of The L. EASL clinical practice guidelines: Management of chronic hepatitis B virus infection. J Hepatol. 2012;57(1):167-85.

6. European Association For The Study Of The L. EASL Clinical Practice Guidelines: management of chronic hepatitis B. J Hepatol. 2009;50(2):227-42.

7. Liaw YF, Leung N, Kao JH, Piratvisuth T, Gane E, Han KH, et al. Asian-Pacific consensus statement on the management of chronic hepatitis B: a 2008 update. Hepatol Int. 2008;2(3):263-83.

8. Martinez-Noguera A, Montserrat E, Torrubia S, Villalba J. Doppler in hepatic cirrhosis and chronic hepatitis. Semin Ultrasound CT MR. 2002;23(1):19-36.

9. Fung J, Lai CL, Chan SC, But D, Seto WK, Cheng C, et al. Correlation of liver stiffness and histological features in healthy persons and in patients with occult hepatitis $\mathrm{B}$, chronic active hepatitis $\mathrm{B}$, or hepatitis B cirrhosis. Am J Gastroenterol. 2010;105(5):1116-22.

10. Zeng MD, Lu LG, Mao YM, Qiu DK, Li JQ, Wan MB, et al. Prediction of significant fibrosis in HBeAg-positive patients with chronic hepatitis B by a noninvasive model. Hepatology. 2005;42(6):143745.

11. Seto WK, Lee CF, Lai CL, Ip PP, Fong DY, Fung J, et al. A new model using routinely available clinical parameters to predict significant liver fibrosis in chronic hepatitis B. PLoS One. 2011;6(8):e23077.
12. Zhang YG, Wang BE, Wang TL, Ou XJ. Assessment of hepatic fibrosis by transient elastography in patients with chronic hepatitis B. Pathol Int. 2010;60(4):284-90.

13. World Health Organization.. Guidelines for the prevention, care and treatment of persons with chronic hepatitis B infection. 2015. Available from: http://www.who.int/entity/hiv/pub/hepatitis/ hepatitis-b-guidelines/en.

14. Jin W, Lin Z, Xin Y, Jiang X, Dong Q, Xuan S. Diagnostic accuracy of the aspartate aminotransferase-to-platelet ratio index for the prediction of hepatitis B-related fibrosis: a leading meta-analysis. BMC Gastroenterol. 2012;12:14.

15. Shoaei SD, Sali S, Karamipour M, Riahi E. Non-invasive histologic markers of liver disease in patients with chronic hepatitis B. Hepat Mon. 2014;14(2):e14228.

16. Castera L. Noninvasive methods to assess liver disease in patients with hepatitis B or C. Gastroenterology. 2012;142(6):1293-1302 e4.

17. Cheng PN, Tsai HW, Chiu YC, Ho CH, Wu IC, Chang TT. Clinical significance of serum HBsAg levels and association with liver histology in HBeAg positive chronic hepatitis B. J Clin Virol. 2013;57(4):323-30.

18. Kim YJ, Cho HC, Choi MS, Lee JH, Koh KC, Yoo BC, et al. The change of the quantitative HBsAg level during the natural course of chronic hepatitis B. Liver Int. 2011;31(6):817-23.

19. Seto WK, Wong DK, Fung J, Ip PP, Yuen JC, Hung IF, et al. High hepatitis B surface antigen levels predict insignificant fibrosis in hepatitis B e antigen positive chronic hepatitis B. PLoS One. 2012;7(8):e43087.

20. Xun YH, Zang GQ, Guo JC, Yu XL, Liu H, Xiang J, et al. Serum hepatitis B surface antigen quantification as a useful assessment for significant fibrosis in hepatitis B e antigen-positive hepatitis B virus carriers. J Gastroenterol Hepatol. 2013;28(11):1746-55.

21. Martinot-Peignoux M, Carvalho-Filho R, Lapalus M, Netto-Cardoso AC, Lada O, Batrla R, et al. Hepatitis B surface antigen serum level is associated with fibrosis severity in treatment-naive, e antigen-positive patients. J Hepatol. 2013;58(6):1089-95.

22. Hong MZ, Huang WQ, Min F, Xu JC, Lin Z, Fang KN, et al. Enhanced HBsAg synthesis correlates with increased severity of fibrosis in chronic hepatitis B patients. PLoS One. 2014;9(1):e87344.

23. Wai CT, Greenson JK, Fontana RJ, Kalbfleisch JD, Marrero JA, Conjeevaram HS, et al. A simple noninvasive index can predict both significant fibrosis and cirrhosis in patients with chronic hepatitis C. Hepatology. 2003;38(2):518-26.

24. Desmet VJ, Gerber M, Hoofnagle JH, Manns M, Scheuer PJ. Classification of chronic hepatitis: diagnosis, grading and staging. Hepatology. 1994;19(6):1513-20.

25. Liu XD, Wu JL, Liang J, Zhang T, Sheng QS. Globulin-platelet mode predicts minimal fibrosis and cirrhosis in chronic hepatitis B virus infected patients. World J Gastroenterol. 2012;18(22):2784-92.

26. Schmilovitz-Weiss H, Tovar A, Halpern M, Sulkes J, Braun M, Rotman Y, et al. Predictive value of serum globulin levels for the extent of hepatic fibrosis in patients with chronic hepatitis B infection. J Viral Hepat. 2006;13(10):671-7.

27. Kim BK, Kim do Y, Park JY, Ahn SH, Chon CY, Kim JK, et al. Validation of FIB-4 and comparison with other simple noninvasive indices for predicting liver fibrosis and cirrhosis in hepatitis $\mathrm{B}$ virus-infected patients. Liver Int. 2010;30(4):546-53.

28. Wang H, Xue L, Yan R, Zhou Y, Wang MS, Cheng MJ, et al. Comparison of FIB-4 and APRI in Chinese HBV-infected patients with persistently normal ALT and mildly elevated ALT. J Viral Hepat. 2013;20(4):e3-10.

29. Shin WG, Park SH, Jang MK, Hahn TH, Kim JB, Lee MS, et al. Aspartate aminotransferase to platelet ratio index (APRI) can predict liver fibrosis in chronic hepatitis B. Dig Liver Dis. 2008;40(4):267-74.

30. Zhu X, Wang LC, Chen EQ, Chen XB, Chen LY, Liu L, et al. Prospective evaluation of FibroScan for the diagnosis of hepatic fibrosis compared with liver biopsy/AST platelet ratio index and FIB-4 in patients with chronic HBV infection. Dig Dis Sci. 2011;56(9):2742-9.

31. Zeng DW, Liu YR, Dong J, Zhu YY, Li YB, Chen J, et al. Serum HBsAg and $\mathrm{HBeAg}$ levels are associated with liver pathological stages in the immune clearance phase of hepatitis B virus chronic infection. Mol Med Rep. 2015;11(5):3465-72. 
32. Pollicino T, Amaddeo G, Restuccia A, Raffa G, Alibrandi A, Cutroneo $\mathrm{G}$, et al. Impact of hepatitis B virus (HBV) pres/S genomic variability on HBV surface antigen and HBV DNA serum levels. Hepatology. 2012;56(2):434-43.

33. Nguyen T, Thompson AJ, Bowden S, Croagh C, Bell S, Desmond PV, et al. Hepatitis B surface antigen levels during the natural history of chronic hepatitis B: a perspective on Asia. J Hepatol. 2010;52(4):508-13.

34. Liang LB, Zhu X, Yan LB, Du LY, Liu C, Chen LY, et al. Serum hepatitis $B$ surface antigen titer and transient elastography in screening for insignificant fibrosis in HBeAg-positive chronic hepatitis B patients. Ther Clin Risk Manag. 2015;11:229-35. 